\title{
Erratum to: Mobile source of high-energy single-cycle terahertz pulses
}

\author{
A. G. Stepanov $\cdot$ S. Henin $\cdot$ Y. Petit $\cdot$ \\ L. Bonacina $\cdot$ J. Kasparian $\cdot$ J.-P. Wolf
}

Received: 26 June 2013 / Accepted: 14 August 2013/Published online: 4 April 2014

(C) Springer-Verlag Berlin Heidelberg 2014

\section{Erratum to: Appl Phys B (2010) 101:11-14 DOI 10.1007/s00340-010-4186-4}

In 2010, we reported [1] on a demonstration of a mobile source of high-energy near-single-cycle $\mathrm{THz}$ pulses based on a tilted pulse front optical rectification set-up, pumped by a mobile terawatt laser (Teramobile). The absolute value of the $\mathrm{THz}$ pulse energy was measured with a pyroelectric detector (Coherent Molectron J4-05). The company does not provide a spectral sensitivity dependence for this detector below $3.3 \mathrm{THz}$. In the reported experiments, generated THz pulses had broad spectra centred at $0.2 \mathrm{THz}$ [1]. Previously, this detector was used for measuring the energy of near-single-cycle $\mathrm{THz}$ pulses with an average frequency of $0.9 \mathrm{THz}$ generated by an accelerator-based source [2]. Afterwards, it was reported that the sensitivity of the Coherent pyroelectric detector at $1 \mathrm{THz}$ was approximately 1.8 times less than the specified sensitivity at $1.06 \mu \mathrm{m}$ [3], and this difference has been take into account in a following publication [7].

The online version of the original article can be found under doi:10.1007/s00340-010-4186-4.

A. G. Stepanov $(\bowtie) \cdot$ S. Henin · Y. Petit · L. Bonacina ·

J. Kasparian · J.-P. Wolf

GAP-Biophotonics, Université de Genève, Chemin de Pinchat

22, 1211 Geneva 4, Switzerland

e-mail: andrey.stepanov@unige.ch

J.-P. Wolf

e-mail: jean-pierre.wolf@unige.ch

Present Address:

Y. Petit

CNRS, University Bordeaux, ICMCB, UPR 9048, 33600 Pessac,

France
Recently, we compared the sensitivity of the Coherent Molectron J4-05 detector with that of another widely used pyroelectric $\mathrm{THz}$ detector (Microtech Instruments) [3-6]. The latter detector is provided with a calibration curve of the spectral dependence of the sensitivity in the frequency range of $0.12-3 \mathrm{THz}$. As a result, we found that at frequencies of $0.2-0.5 \mathrm{THz}$, the Coherent Molectron J4-05 detector underestimates the $\mathrm{THz}$ pulse energy by a factor of about 3.5 with regard to the Microtech Instruments detector. This finding evidences an additional drop of the Coherent Molectron J4-05 detector sensitivity for $\mathrm{THz}$ wave frequencies below $1 \mathrm{THz}$. By this comparison, we conclude that the THz pulse energy in Ref. [1] should significantly be re-estimated, up to $175 \mu \mathrm{J}$ (instead of the initially published value of $50 \mu \mathrm{J}$ ), which is to the best of our knowledge the highest energy of near-single-cycle $\mathrm{THz}$ pulses generated by tabletop sources.

\section{References}

1. A.G. Stepanov, S. Henin, Y. Petit, L. Bonacina, J. Kasparian, J.-P. Wolf, Appl. Phys. B 101, 11 (2010)

2. Y. Shen, T. Watanabe, D.A. Arena, C.-C. Kao, J.B. Murphy, T.Y. Tsang, X.J. Wang, G.L. Carr, Phys. Rev. Lett. 99, 043901 (2007)

3. F. Blanchard, L. Razzari, H.-C. Bandulet, G. Sharma, R. Morandotti, J.-C. Kieffer, T. Ozaki, M. Reid, H.F. Tiedje, H.K. Haugen, F.A. Hegmann, Opt. Express 15, 13212 (2007)

4. A.G. Stepanov, L. Bonacina, S.V. Chekalin, J.-P. Wolf, Opt. Lett. 33, 2497 (2008)

5. H. Hirori, A. Doi, F. Blanchard, K. Tanaka, Appl. Phys. Lett. 98, $091106(2011)$

6. J.A. Fülöp, L. Pálfalvi, S. Klingebiel, G. Almási, F. Krausz, S. Karsch, J. Hebling, Opt. Lett. 37, 557 (2012)

7. F. Blanchard, G. Sharma, X. Ropagnol, L. Razzari, R. Morandotti, T. Ozaki, Opt. Expres 17, 6044 (2009) 